Zbl Arbeitsmed 2021 $\cdot 71: 19-23$ https://doi.org/10.1007/s40664-020-00400-y Online publiziert: 6 . Juli 2020

(c) Der/die Autor(en) 2020

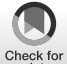

\author{
R. Strametz ${ }^{1,2} \cdot$ H. Rösner' $\cdot$ M. Ablöscher ${ }^{3,4} \cdot$ W. Huf ${ }^{3,4} \cdot$ B. Ett| $\left.\right|^{3,4} \cdot$ M. Raspe \\ 'Wiesbaden Business School, Hochschule RheinMain, Wiesbaden, Deutschland \\ ${ }^{2}$ Aktionsbündnis Patientensicherheit e.V., Berlin, Deutschland \\ ${ }^{3}$ Karl Landsteiner Institut für klinisches Risikomanagement, Wien, Österreich \\ ${ }^{4}$ Klinik Hietzing, Wiener Gesundheitsverbund, Wien, Österreich \\ ${ }^{5}$ Medizinische Klinik m. S. Infektiologie und Pneumologie, Charité - Universitätsmedizin Berlin, Berlin \\ Deutschland
}

\title{
Entwicklung und Validation eines Fragebogens zur Beurteilung der Inzidenz und Reaktionen von Second Victims im Deutschsprachigen Raum (SeViD)
}

in englischsprachigen Ländern für Second Victims eingeführt [14, 18], die sowohl Vorteile im Hinblick auf medizinische $[14,15]$ und ökonomische Folgen [16] zeigen. In Deutschland formulierte die Deutsche Gesetzliche Unfallversicherung Handlungsempfehlungen für die Behandlung von Mitarbeitern nach traumatischen Ereignissen [17]. Obwohl psychologische Unterstützung für Mitarbeiter nach traumatischen Ereignissen in anderen Bereichen wie dem Luft- und Schienenverkehr $[19,20]$ bereits vorhanden sind, gab es bislang noch keine systematische Bewertung des Phänomens für den Bereich des deutschen Gesundheitssystems. Aus diesem Grund wurde das Projekt SeViD (Second Victims im Deutschsprachigen Raum) gestartet. In einem ersten Schritt des Projekts wurde ein deutschsprachiger Fragebogen entwickelt und validiert, der berufsgruppenunabhängig die Inzidenz von Second-Victim-Traumatisierung, die selbstbeobachtete Verarbeitung der Traumatisierung sowie eine Bewertung von Unterstützungsmaßnahmen erfragt. Dies soll sowohl den Status quo von Second-Victim-Traumatisierungen im deutschsprachigen Raum dokumentieren als auch eine Evaluation von Unterstützungsprogrammen im Sinne eines Vorher-Nachher-Vergleichs ermöglichen. Verbunden ist diese laufende Arbeit mit dem Ziel, die negativen Auswirkungen von SecondVictim-Erfahrungen zu minimieren.

\section{Methoden}

Eine systematische Literaturrecherche in MEDLINE und Google Scholar wurde durchgeführt, um Fragebögen zu identifizieren, die zuvor entwickelt und/oder genutzt wurden, um das Second-Victim-Phänomen im Gesundheitswesen $\mathrm{zu}$ evaluieren. Dabei wurde die Suche auf englisch- sowie deutschsprachige Publikationen beschränkt. Basierend auf allen identifizierten Fragebögen, wurde die deutschsprachige Version des Fragebogens aus Elementen etablierter Befragungsinstrumente zusammengestellt. Damit eine Vergleichbarkeit $\mathrm{zu}$ Befragungen in anderen medizinischen Fachgebieten sichergestellt werden kann, wird durch den Fragebogen keine spezielle medizinische Berufsgruppe gesondert adressiert, sondern es wurden lediglich demographische Faktoren angepasst. Um eine Akzeptanz des Fragebogens, insbesondere unter Ärztinnen und Ärzten, zu erlangen, wurde darauf geachtet, dass die Bearbeitungsdauer des Fragebogens einen Zeitraum von 5 min nicht überschreitet. Für den Fall, dass keine Second-Victim-Erfahrung ramme für medizinisches Fachpersonal 


\begin{tabular}{|c|c|c|c|}
\hline Erstautor/in & $\begin{array}{l}\text { Name des Fra- } \\
\text { gebogens/der } \\
\text { Studie }\end{array}$ & Setting/Studienpopulation & $\begin{array}{l}\text { Ähnliche Pu- } \\
\text { blikation oder } \\
\text { andere identifi- } \\
\text { zierte Quelle }\end{array}$ \\
\hline $\begin{array}{l}\text { Burlison, JD } \\
{[26]}\end{array}$ & $\begin{array}{l}\text { Second Victim } \\
\text { Experience and } \\
\text { Support Survey } \\
\text { (SVEST) }\end{array}$ & $\begin{array}{l}\text { Mitarbeitende einer Kinderklinik, die Kinder mit schwerer Krankheit behandeln, u. a. Pfle- } \\
\text { gepersonal, Ärzteschaft, Apotheke, technisches Personal etc. }\end{array}$ & \\
\hline $\begin{array}{l}\text { Edrees, } \mathrm{HH} \\
{[28]}\end{array}$ & $\begin{array}{l}\text { Second Victim } \\
\text { Questionnaire }\end{array}$ & $\begin{array}{l}350 \text { medizinische Fachpersonen aus verschiedenen Fachrichtungen, die an der Session } \\
\text { "Healthcare Workers: the ,Second Victims"' des „Johns Hopkins Medicine 1st Annual Pa- } \\
\text { tient Safety Summit" teilnahmen }\end{array}$ & {$[28,29]$} \\
\hline $\begin{array}{l}\text { Gazoni, FM } \\
\text { [27] }\end{array}$ & $\begin{array}{l}\text { Perioperative } \\
\text { Catastrophes } \\
\text { Survey }\end{array}$ & $\begin{array}{l}\text { Postalische Befragung eine Stichprobe von } 1200 \text { zufällig ausgewählten Mitgliedern der } \\
\text { American Society of Anesthesiologists }\end{array}$ & \\
\hline Scott, SD [8] & $\begin{array}{l}\text { The Second Victim } \\
\text { Experience Survey }\end{array}$ & $\begin{array}{l}\text { Online-Fragebogen mit } 10 \text { Items für ca. } 5300 \text { Mitarbeitende im Gesundheitsdienst der } \\
\text { University of Missouri }\end{array}$ & [28] \\
\hline $\begin{array}{l}\text { Waterman, } \\
\mathrm{AD}[10]\end{array}$ & n.a. & $\begin{array}{l}\text { Kombinierte anonymisierte postalische Befragung oder Online-Befragung klinisch tätiger } \\
\text { Ärztinnen und Ärzte aus den Disziplinen Innere Medizin, Chirurgie (Allg. Chirurgie und alle } \\
\text { Fachgebiete) und Allgemeinmedizin in } 13 \text { Krankenhäusern in den USA und einer repräsen- } \\
\text { tativen Stichprobe } 2400 \text { kanadischer Ärztinnen und Ärzte (Innere Medizin/Chirurgie) } \\
\text { Online-Fragebogen für Kollegen und Mitglieder des Royal College of Physicians (RCP) }\end{array}$ & [26] \\
\hline Unbekannt & $\begin{array}{l}\text { The Second Victim } \\
\text { Experience Survey }\end{array}$ & Online Fragebogen, derzeit open access abrufbar & {$[18,27,28]$} \\
\hline
\end{tabular}

vorliegt, sollte somit ein akzeptabler Rücklauf gewährleistet sein und Selektionseffekte minimiert werden. Da die Fragebogenerstellung auf etablierte Befragungsinstrumente aus dem angloamerikanischen Raum zurückgriff, wurde der Fokus der Validierung auf die Überprüfung der Inhaltsvalidität gelegt, da diese durch Übersetzungseffekte möglicherweise beeinträchtigt wird. Dies erfolgte anhand einer vorläufigen Version des Fragebogens mittels kognitiver Pretest-Techniken. Dabei wurden Methoden wie "paraphrasing“, "probing“ und „thinking aloud“ [21] genutzt.

Ein Pretest wurde nach Einverständniserklärung durch Freiwillige der Medizinischen Klinik mit Schwerpunkt Infektiologie und Pneumologie der Charité - Universitätsmedizin Berlin und des Krankenhauses Hietzing mit Neurologischem Zentrum Rosenhügel des Wiener Krankenanstaltenverbundes durchgeführt. Es wurde medizinisches Fachpersonal aus verschiedenen Berufsgruppen einbezogen (Ärzteschaft, Krankenpflege, Angehörige medizinisch therapeutischer und diagnostischer Berufe), unabhängig davon, ob die Person zuvor schon einmal die Rolle eines Second Victims eingenommen hatte oder nicht. Der Stichprobenumfang wurde basierend auf Empfehlungen von Willis [22] und Lenzner [21] auf ein Minimum von 15 Freiwilligen festgelegt. Kognitive Pretests wurden durch einen unbekannten und von beiden Krankenhäusern unabhängigen Forscher durchgeführt, um einem möglichen Observationsbias vorzubeugen. Dies erfolgte anhand eines strukturierten Interview-Leitfadens, der insbesondere aktiv das Verständnis des Begriffs Second Victim, die Vollständigkeit möglicher auslösender Faktoren einer entsprechenden Traumatisierung, das Verständnisses möglicher Effekte einer Traumatisierung sowie der Akzeptanz aller Items sowie des Befragungsinstruments als Ganzes erfragte. Alle Fragebögen waren zur Sicherung des Datenschutzes komplett anonymisiert. In allen Fragebögen wurde die anonyme Nutzung der Daten für den wissenschaftlichen Gebrauch angekündigt und das Einverständnis zur Nutzung der Daten eingeholt.

\section{Ergebnisse}

Aus insgesamt 9 relevanten Quellen, die sich möglicherweise als geeignet für einen partiellen Einbezug in den vorläufigen Fragebogen zeigten, konnten 6 Fragebögen identifiziert werden $[6,8$,
18, 24-29]. Details zu einbezogenen Fragebögen sind in $\bullet$ Tab. 1 aufgezeigt. Der erste Entwurf des Fragebogens bestand neben den demographischen Angaben aus den 3 Bereichen „Allgemeine Erfahrungen mit dem Phänomen“, „Second Victim Symptome“ und „Second Victim Unterstützungsstrategien " und wurde auf 40 Fragen limitiert, die aus etablierten Fragebögen übernommen oder angepasst wurden, wie in - Tab. 2 gezeigt wird. Fünfzehn medizinische Fachpersonen (Ärzteschaft, Krankenpflege, Angehörige medizinisch therapeutischer und diagnostischer Berufe) der Charité Berlin, $(n=6)$ und des Krankenhauses Hietzing, Wien $(n=9)$ nahmen an den Pretests des vorläufigen Fragebogens teil.

Basierend auf den kognitiven Pretests wurden 7 Fragen in allen 3 Bereichen leicht modifiziert. Allen Teilnehmenden war es möglich, ausgewählte Fragen sinngemäß korrekt neu zu formulieren oder Fachtermini, insbesondere den Begriff Second Victim, korrekt zu umschreiben, unabhängig davon, ob vor unserem Pretest das Phänomen Second Victim als solches bekannt war oder nicht. Die durchschnittliche Bearbeitungszeit des Fragebogens belief sich auf 9:01 $( \pm 3: 05) \mathrm{min}$ beivorherigerSecond-Victim-Erfahrung bzw. auf 4:19 $( \pm 0: 59)$ min ohne vorhe- 
Zbl Arbeitsmed 2021 · 71:19-23 https://doi.org/10.1007/s40664-020-00400-y

(c) Der/die Autor(en) 2020

R. Strametz · H. Rösner · M. Ablöscher · W. Huf · B. Ettl · M. Raspe

\section{Entwicklung und Validation eines Fragebogens zur Beurteilung der Inzidenz und Reaktionen von Second Victims im Deutschsprachigen Raum (SeViD)}

\section{Zusammenfassung}

Einleitung. Second Victims, definiert als medizinische Fachpersonen, die durch einen unvorhergesehenen klinischen Zwischenfall traumatisiert werden, sind ein verbreitetes Phänomen im Gesundheitswesen. Studien aus den USA zeigen hohe Inzidenzen zwischen 10 und $42 \%$ bei medizinischem Personal. Allerdings existiert kein validierter deutscher Fragebogen, der sich mit der Inzidenz und den Auswirkungen auseinandersetzt. Deshalb wurde im Rahmen des SeViD-Projekts (Second Victims im Deutschsprachigen Raum) ein deutschsprachiger Fragebogen zur Beurteilung von Second-Victim-Erfahrungen entwickelt.

Methoden. Basierend auf einer Literaturrecherche nach existierenden Fragebögen in englischer Sprache wurde eine vorläufige
Version des Fragebogens bestehend aus 4 Bereichen und 14 Fragen erstellt. Diese wurde kognitiven Pretests unterzogen, um die Inhaltsvalidität zu überprüfen.

Ergebnisse. Fünfzehn medizinische Fachpersonen aus Krankenhäusern in Deutschland $(n=6)$ und Österreich $(n=9)$ nahmen nach Einverständniserklärung als Freiwillige an allen Pretests teil. Sieben Fragen in 3 Bereichen wurden basierend auf kognitiven Pretests leicht modifiziert. Die durchschnittliche Bearbeitungszeit des Fragebogens betrug 9:01 $( \pm 3: 05) \mathrm{min}$ im Fall einer vorherigen SecondVictim-Erfahrung und 4:19 ( $\pm 0: 59)$ min ohne entsprechendes Erlebnis. Die Befragung wurde von allen Freiwilligen als angemessen bewertet.
Schlussfolgerung. Es konnte ein inhaltsvalidierter Fragebogen entwickelt werden, der die Auswirkung des Second-Victim-Phänomens in stationären Gesundheitseinrichtungen im deutschsprachigen Raum beurteilen kann. Dieser Fragebogen soll in verschiedenen Settings für medizinisches Fachpersonal sowohl für Erhebungen des Status quo als auch für Verlaufsbeobachtungen in Interventionsstudien eingesetzt werden.

Schlüsselwörter

Patientensicherheit · Medizinisches Fachpersonal · Posttraumatische Belastungsstörung · Klinisches Risikomanagement . Literaturrecherche

\section{Development and validation of a questionnaire to assess incidence and reactions of second victims in German-speaking countries (SeViD)}

\section{Abstract}

Background. Second victims, defined as healthcare team members traumatized by an unanticipated clinical incident, are a common phenomenon in healthcare systems. Studies from the USA showed a high incidence among medical personnel of between $10 \%$ and $42 \%$; however, no validated German questionnaire for assessing the incidence and impact exists. Therefore, within the framework of the second victims in German-speaking countries (SeViD) project a German language questionnaire for assessment of second victim experiences was developed.

Methods. Based on a literature search of available questionnaires in the English language, a preliminary version of the questionnaire consisting of 4 domains and 14 questions was constructed. This version was subjected to cognitive pretesting to examine the content validity.

Results. In this study 15 healthcare professionals from hospitals in Germany $(n=6)$ and Austria $(n=9)$ participated as volunteers for all pretests after providing informed consent. A total of seven questions in three domains were slightly modified based on cognitive pretests. The mean time for completion of this questionnaire was 9:01 $\mathrm{min}( \pm 3: 05 \mathrm{~min})$ in the case of a previous second victim experience and 4:19 $\mathrm{min}( \pm 0: 59 \mathrm{~min})$ with no such experience. The questionnaire was deemed to be appropriate by all volunteers.
Conclusion. A validated questionnaire could be developed, which can be used to assess the impact of the second victim phenomenon in inpatient healthcare facilities in the Germanspeaking area. This questionnaire will be implemented in various settings for healthcare professionals for the compilation of the status quo and for observation of the course in interventional studies.

Keywords

Patient safety - Medical personnel · Posttraumatic stress disorder - Clinical risk management $\cdot$ Literature search rige Erfahrung, was von allen Freiwilligen hinsichtlich der Dauer als akzeptabel angesehen wurde. Keine Frage wurde als unangemessen bezeichnet. Ebenso wurde keine wichtige Information im Fragebogen auch auf Nachfrage durch die Teilnehmenden vermisst.

\section{Diskussion}

Im Rahmen des vorliegenden Projekts konnte ein Fragebogen entwickelt und inhaltlich validiert werden, der die Erfahrung von Second Victims im Gesundheitswesen in deutschsprachigen Ländern bewertet und von allen partizipierenden Freiwilligen während kognitiver Pretests als angemessen angesehen wurde. Obwohl eine kleine Untergruppe an
Teilnehmern für Pretests immer mit dem Risiko eines Selektionsbias oder eines Hawthorne-Effekts assoziiert sein kann, zeigten die Reaktionen von teilnehmenden Freiwilligen mit vorherigen SecondVictim-Erfahrungen keinen Hinweis auf einen Observationsbias. Die Auswahl der Fragen basiert auf zuvor entwickelten, etablierten englischen Fragebögen. Dies unterstützt die Inhaltsvalidität zusätzlich. Die Veränderungen der vorläufigen 
Tab. 2 Liste der Bereiche und Items des SeViD-Fragebogens (Second Victims im Deutschsprachigen Raum)

\begin{tabular}{l} 
Bereich \\
\hline Allgemeine \\
Erfahrung mit \\
dem Phänomen
\end{tabular}

Second Victim Symptome

Second Victim Unterstützungsstrategien

Item

Kenntnis des Begriffs Second Victim

Lebensprävalenz der Second-Victim-Erfahrung

12-Monats-Prävalenz der Second-Victim-Erfahrung

Art des Schlüsselereignisses

Streben nach Unterstützung nach einem Schlüsselereignis

Art der Unterstützungsgruppe nach einem Schlüsselereignis

Eigen wahrgenommene Zeit bis zur vollkommenen Erholung von einem Schlüsselereignis

Angst vor Verlust des Arbeitsplatzes

Antriebslosigkeit

Depressive Stimmung

Konzentrationsschwierigkeiten

Wiedererleben der Situation außerhalb der beruflichen Tätigkeit

Wiedererleben der Situation in ähnlichen beruflichen Situationen

Aggressives, bewusst risikohaftes Verhalten

Defensives, übervorsichtiges Verhalten

Psychosomatische Reaktionen (Kopf-, Rückenschmerzen etc.)

Schlafstörungen oder übermäßiges Schlafbedürfnis

Gebrauch von Alkohol/Drogen aufgrund des Ereignisses

Schamgefühle

Schuldgefühle

Selbstzweifel

Soziale Isolation

Wut/Zorn gegenüber anderen

Wut/Zorn gegenüber mir

Wunsch nach Unterstützung durch andere

Wunsch, das Ereignis aufzuarbeiten, um es besser zu verstehen das Ereignis zu verarbeiten
Angst vor Ausgrenzung durch Kollegen

Die Möglichkeit, von der Tätigkeit direkt eine Auszeit nehmen zu können, um

Zugang zu professioneller Seelsorge bzw. psychologischem oder psychiatrischem Dienst vor Ort

Die Möglichkeit, meine emotionalen bzw. ethischen Gedanken zu diskutieren

Klare und zeitnahe Information bezüglich des Ablaufs nach einem ernsthaften Ereignis (z. B. Schadenfallanalyse, Anfertigung eines Fehlerberichts)

Formale emotionale Unterstützung im Sinne einer organisierten kollegialen Hilfe

Informelle emotionale Unterstützung

Schnelle Aufarbeitung („,debriefing") der Situation bzw. schnelle Krisenintervention (im Team oder individuell)

Unterstützung/Mentoring bei Fortsetzung der patientennahen Tätigkeit

Unterstützung bei der Kommunikation mit Patienten und/oder Angehörigen

Vorgaben bezüglich der von mir erwarteten Rolle bzw. Tätigkeiten bei einem ernsthaften Ereignis

Unterstützung, um aktiv am Aufarbeitungsprozess dieses Ereignisses mitzuwirken

Eine sichere Möglichkeit, um Informationen beizusteuern, die helfen sollen, ähnliche Ereignisse in Zukunft zu vermeiden

Die Möglichkeit der Inanspruchnahme rechtlicher Beratung nach einem schwerwiegenden Fehler
Version waren gering und wurden wie erwartet überwiegend durch Übersetzungseffekte existierender Fragen ins Deutsche verursacht. Eine quantitative Auswertung mit entsprechend angemessener Fallzahl wird im Rahmen der ersten Erhebung im deutschsprachigen Raum (SeViD-I-Studie) erwartet. Dabei werden erstmals neben Fragen der Inzidenz und Verarbeitung des Phänomens sowohl von Second Victims als auch von nichttraumatisierten Behandelnden Einstellungen $\mathrm{zu}$ möglichen Unterstützungsangeboten erhoben, sodass es ggf. möglich ist, systematische Unterschiede in der Einschätzung der Sinnhaftigkeit von Hilfsmaßnahmen in Abhängigkeit persönlicher Vorerfahrungen zu identifizieren.

\section{Fazit für die Praxis}

- Ein inhaltsvalidierter Fragebogen wurde entwickelt, welcher die Auswirkungen des Second-Victim-Phänomens in stationären Gesundheitseinrichtungen im deutschsprachigen Raum erhebt.

- Dieser Fragebogen soll in verschiedenen Settings für medizinisches Fachpersonal - sowohl für Erhebungen des Status quo als auch für Verlaufsbeobachtungen im Rahmen kommender Interventionsstudien eingesetzt werden.

\section{Korrespondenzadresse}

Prof. Dr. med. R. Strametz

Wiesbaden Business School, Hochschule RheinMain

Bleichstr. 44, 65183 Wiesbaden, Deutschland strametz@aps-ev.de

Danksagung. Die Autoren danken allen freiwilligen Teilnehmenden, die an der Validation dieses Fragebogens teilgenommen haben.

Funding. Open Access funding provided by Projekt DEAL.

\section{Einhaltung ethischer Richtlinien}

Interessenkonflikt. R. Strametz, H. Rösner, M. Ablöscher, W. Huf, B. Ettl und M. Raspe geben an, dass kein Interessenkonflikt besteht. 
Aufgrund des Studiendesigns war keine formale $A b-$ stimmung einer Ethikkommission erforderlich. Zur Gewährleistung der Datensicherheit wurden Daten ohne jegliche demografischen Informationen erhoben, die eine Identifikation von Teilnehmenden erlaubt hätten. Alle Teilnehmenden gaben ihr Einverständnis zur Teilnahme an der Studie. Einverständnis der Veröffentlichung: Alle Teilnehmenden wurden über die Studie informiert und gaben ihre Einwilligung zur Veröffentlichung der Studiendaten.

Open Access Dieser Artikel wird unter der Creative Commons Namensnennung 4.0 International Lizenz veröffentlicht, welche die Nutzung, Vervielfältigung, Bearbeitung, Verbreitung und Wiedergabe in jeglichem Medium und Format erlaubt, sofern Sie den/die ursprünglichen Autor(en) und die Quelle ordnungsgemäß nennen, einen Link zur Creative Commons Lizenz beifügen und angeben, ob Änderungen vorgenommen wurden.

Die in diesem Artikel enthaltenen Bilder und sonstiges Drittmaterial unterliegen ebenfalls der genannten Creative Commons Lizenz, sofern sich aus der Abbildungslegende nichts anderes ergibt. Sofern das betreffende Material nicht unter der genannten Creative Commons Lizenz steht und die betreffende Handlung nicht nach gesetzlichen Vorschriften erlaubt ist, ist für die oben aufgeführten Weiterverwendungen des Materials die Einwilligung des jeweiligen Rechteinhabers einzuholen.

Weitere Details zur Lizenz entnehmen Sie bitte der Lizenzinformation auf http://creativecommons.org/ licenses/by/4.0/deed.de.

\section{Literatur}

1. Michaelis M, Hofmann FM, Stößel U, Hofmann F (2017) Norovirusausbrüche und Kooperation zwischen Hygienepersonal und Betriebsärzten im Krankenhaus. Zentralbl Arbeitsmed 67:309-313. https://doi.org/10.1007/s40664-017-0206-0

2. Quart J, Deutsch T, Carmienke S, Döpfmer S, Frese T (2018) Willingness to commute among future physicians: a multicenter cross-sectional survey of German medical students. J Occup Med Toxicol 13:17.https://doi.org/10.1186/s12995-018-02002

3. Strametz R, Schneider T, Pitz A, Raspe M (2019) Survival-Day @ Wiesbaden business school-evaluation of a short-term educational intervention to reduce work-associated health risks during nursing internships of students in health care economics. J Occup Med Toxicol 14:30. https://doi.org/10.1186/s12995-019-0251-z

4. Appel P, Schuler M, Vogel H, Oezelsel A, Faller H (2017) Short Questionnaire for Workplace Analysis (KFZA): Factorial validation in physicians and nurses working in hospital settings. J Occup Med Toxicol 12:11. https://doi.org/10.1186/s12995017-0157-6

5. Brütting $M$, Hermanns I, Nienhaus $A$, Ellegast $R$ (2017) Muskel-Skelett-Belastungen beim Schieben und Ziehen von Krankenbetten und Rollstühlen. Zentralbl Arbeitsmed 67:64-77. https://doi. org/10.1007/s40664-016-0150-4

6. Scott SD, Hirschinger LE, Cox KR, McCoig M, Brandt J, Hall LW (2009) The natural history of recovery for the healthcare provider "second victim" after adverse patient events. Qual Saf
Health Care 18:325-330. https://doi.org/10.1136/ qshc.2009.032870

7. Wu AW (2000) Medical error: the second victim BMJ 320:726-727. https://doi.org/10.1136/bmj. 320.7237 .726

8. Waterman AD, Garbutt J, Hazel E, Dunagan WC, Levinson W, Fraser VJ, Gallagher TH (2007) The emotional impact of medical errors on practicing physicians in the United States and Canada. Jt Comm J Qual Patient Saf 33:467-476. https://doi org/10.1016/s1553-7250(07)33050-x

9. von Laue N, Schwappach D, Hochreutener M (2012) "Second victim" - Umgang mit der Krise nach dem Fehler. Ther Umsch 69:367-370. https://doi.org/ 10.1024/0040-5930/a000300

10. Schwappach DL, Boluarte TA (2009) The emotional impact of medical error involvement on physicians: a call for leadership and organisational accountability. Swiss Med Wkly 139:9-15

11. Grissinger M (2014) Too many abandon the "second victims" of medical errors. PT 39:591-592

12. West $C P$, Huschka MM, Novotny PJ, Sloan JA, Kolars JC, Habermann TM, Shanafelt TD (2006) Association of perceived medical errors with resident distress and empathy: A prospective longitudinal study. JAMA 296:1071-1078. https:// doi.org/10.1001/jama.296.9.1071

13. Seys D, Wu AW, van Gerven E, Vleugels $A$, Euwema M, Panella M et al (2013) Health care professionals as second victims after adverse events: a systematic review. Eval Health Prof 36:135-162. https://doi.org/10.1177/0163278712458918

14. Edrees H, Connors C, Paine L, Norvell M, Taylor H, Wu AW (2016) Implementing the RISE second victim support programme at the Johns Hopkins Hospital:A case study.BMJOpen 6:e11708.https:// doi.org/10.1136/bmjopen-2016-011708

15. Burlison JD, Quillivan RR, Scott SD, Johnson S, Hoffman JM (2016) The effects of the second victim phenomenon on work-related outcomes: connecting self-reported Caregiver distress to turnover intentions and absenteeism. JPatient Saf. https://doi.org/10.1097/PTS.0000000000000301

16. Moran D, Wu AW, Connors C, Chappidi MR, Sreedhara SK, Selter JH, Padula WV (2017) Costbenefit analysis of a support program for nursing staff. J Patient Saf. https://doi.org/10.1097/PTS. 0000000000000376

17. Deutsche Gesetzliche Unfallversicherung e.V (2017) DGUV Information 206-023: Standards in der betrieblichen psychologischen Erstbetreuung (bpE) bei traumatischen Ereignissen. DGUV Deutsche Gesetzliche Unfallversicherung Spitzenverband, Berlin

18. Scott SD, Hirschinger LE, Cox KR, McCoig M, Hahn-Cover K, Epperly KM et al (2010) Caring for our own: deploying a systemwide second victim rapid response team. Jt Comm J Qual Patient Saf 36:233-240

19. European Parliament and the Council. Regulation (EU) 2016/796 of the European Parliament and of the Council of 11 May 2016on the European Union Agency for Railways and repealing Regulation (EC) No 881/2004: L 138;26. Mai 2016.

20. European Parliament and the Council. Regulation (EU) 2018/1139of the European Parliament and of the Council of 4 July 2018on common rules in the field of civil aviation and establishing a European Union Aviation Safety Agency, and amending Regulations (EC) No 2111/2005, (EC) No 1008/2008, (EU) No 996/2010, (EU) No 376/2014 and Directives 2014/30/EU and 2014/53/EU of the European Parliament and of the Council, and repealing Regulations (EC) No 552/2004 and (EC)
No 216/2008of the European Parliament and of the Council and Council Regulation (EEC) No 3922/91: L212/1;22. Aug. 2018.

21. Lenzner T, Neuert C, Otto W (2016) GESIS survey guidelines. https://www.gesis.org/fileadmin/ upload/SDMwiki/LenznerNeuertOtto_Cognitive_ Pretesting.pdf.Zugegriffen: 7.Jan. 2020

22. Willis GB (2005) Cognitive interviewing: a tool for improving questionnaire design. SAGE, Thousand Oaks

23. Bortz J, Döring N (2015) Forschungsmethoden und Evaluation: Für Human- und Sozialwissenschaftler 5. Aufl. Springer, Berlin

24. Burlison JD, Scott SD, Browne EK, Thompson SG, Hoffman JM (2017) The second victim experience and support tool: validation of an organizational resource for assessing second victim effects and the quality of support resources. J Patient Saf 13:93-102. https://doi.org/10.1097/PTS. 0000000000000129

25. Gazoni FM, Amato PE, Malik ZM, Durieux ME (2012) The impact of perioperative catastrophes on anesthesiologists: results of a national survey. Anesth Analg 114:596-603. https://doi.org/10. 1213/ANE.0b013e318227524e

26. Harrison R, Lawton R, Stewart K (2014) Doctors' experiences of adverse events in secondary care: the professional and personal impact. Clin Med 14:585-590. https://doi.org/10.7861/ clinmedicine.14-6-585

27. The Second Victim Experience Survey. https:// www.surveymonkey.com/r/CaringforOurOwn. Zugegriffen 7.Jan. 2020.

28. Edrees HH, Paine LA, Feroli ER, Wu AW (2011) Health care workers as second victims of medical errors. Pol Arch Intern Med 121:101-108. https://doi.org/ 10.20452/pamw.1033

29. Medically Induced Trauma Support Services (MITSS) (2010) MITSS staff support survey. http:// www.mitsstools.org/uploads/3/7/7/6/3776466/ mitss_staff_support_survey.pdf. Zugegriffen: 7 . Jan. 2020 